\title{
World Antimicrobial Awareness Week 2021 - Spread Awareness, Stop Resistance
}

\author{
Di Wu'; Timothy R. Walsh',; Yongning Wu $\mathbf{W}^{3, *}$
}

The antimicrobial resistance (AMR) Tripartite organizations - the World Health Organization (WHO), the Food and Agriculture Organization of the United Nations (FAO), and the World Organization for Animal Health (Office International des Epizooties, OIE) - are pleased to announce that the theme of World Antimicrobial Awareness Week (WAAW) 2021 will be "Spread Awareness, Stop Resistance" (1). China will participate in the WAAW 2021 and organize the China Antimicrobial Awareness Week (CAAW) 2021.

\section{BACKGROUND}

Celebrated between November 18-24 each year (2), WAAW aims to increase awareness of global AMR and to encourage best practices among the general public, healthcare workers, farmers, animal health professionals, and policymakers to avoid the further emergence and dissemination of drug-resistant infections. In May 2015, a global action plan to tackle AMR was endorsed at the World Health Assembly, supported by FAO and OIE. The first objective of the plan was to "Improve awareness and understanding of antimicrobial resistance through effective communication, education and training." To help achieve this objective, the FAO, OIE, and WHO (collectively known as the Tripartite) have jointly supported WAAW since 2015, together with the general public, students, policymakers, and professionals from various sectors across the world. The overarching slogan of World Antimicrobial Awareness Week continues to be "Antimicrobials: Handle with Care". WAAW is celebrated from November 18-24 every year, endorsing the following campaign objectives: 1) Position AMR as a globally recognized crisis with meaningful engagement across all sectors - human, animal, plant, and environment as the One Health approach, where inappropriate use of antimicrobials in both humans and animals is contributing to the problem and no sector can tackle the problem in isolation; 2) Raise awareness to protect antimicrobial sustainability through prudent and responsible use; 3) Highlight the roles and responsibilities that individuals, governments, professional societies, and organizations in human, animal, environment, and plant health play in addressing and tackling antimicrobial resistance; 4) Encourage behavioral changes that will result in the prudent use of antimicrobials across all relevant sectors and convey the message that simple and sensible actions can make a positive impact.

Tripartite guidance is designed to provide us with the essential information required to participate in the campaign with the hopes that it will help to inspire and imbue us to develop our local public awareness, initiative, and activities. The WAAW 2021 campaign will encourage stakeholders, including policymakers, healthcare providers, the general public, and livestock custodians, to recognize that everyone can be an "AMR Awareness" champion. WAAW 2021 participants are encouraged to disseminate awareness on what AMR is, share stories about its consequences, and demonstrate how the actions of individuals, families, professionals, and communities can have an impact on preventing the spread of AMR. AMR is not a pathogen problem, nor is it a pig or poultry problem, it is a "people problem," and a behavioral change in the demand for and the prescribing of antibiotics is required if the problem is to be effectively tackled. Campaigns to raise awareness, and recognition that a problem exists, are the first step towards the desired behavioral change.

China is a world leader in both human and animal healthcare and its support is essential to making this campaign a success!

\section{Why Address AMR with the One Health Approach?}

AMR occurs when bacteria, viruses, fungi, and parasites change their genetic makeup and, consequently, no longer respond to antimicrobials. AMR makes infections more challenging to treat and eradicate, and it increases the 
risk of disease spread, severe illness, and death. Measures to prevent infection in humans and the subsequent requirement for antibiotics include getting vaccinated, practising safer sex, good hand hygiene, food safety practices, bolstering immune status by improved diet and wellbeing, and increasing availability of safe potable water and sanitation facilities. Historically, in food animal production, breeding decisions were based on selecting for performance traits, like weight gain, food conversion efficiency, egg production, and milk yield, but now the animal geneticists are including disease resistance traits. This coupled with improved husbandry practices, better animal welfare standards, improved nutrition, and the increased use of immunization are reducing the need for antibiotics and the associated risk of developing multidrug resistant zoonotic agents.

AMR is a complex problem affecting human, animal, plant, and environmental health and the impacts of AMR affects all four sectors and there is cross over between them. Microbes can exchange mobile genetic elements conveying disease resistance between each other making tackling this problem challenging. Therefore, addressing AMR requires a holistic and multisectoral approach — referred to as the One Health approach (3-4). This initiative is a collaborative, multisectoral, and trans-disciplinary approach recognizing the interconnections between people, animals, plants, and their shared environment. By designing and implementing multisectoral programs, policies, legislation, and research with professionals from human, terrestrial and aquatic animal and plant health, food and feed production, and the environment, AMR can be more effectively addressed and communicated to achieve better One Health outcomes (Figure 1). Each sector must play its part, and everyone has a role to play.

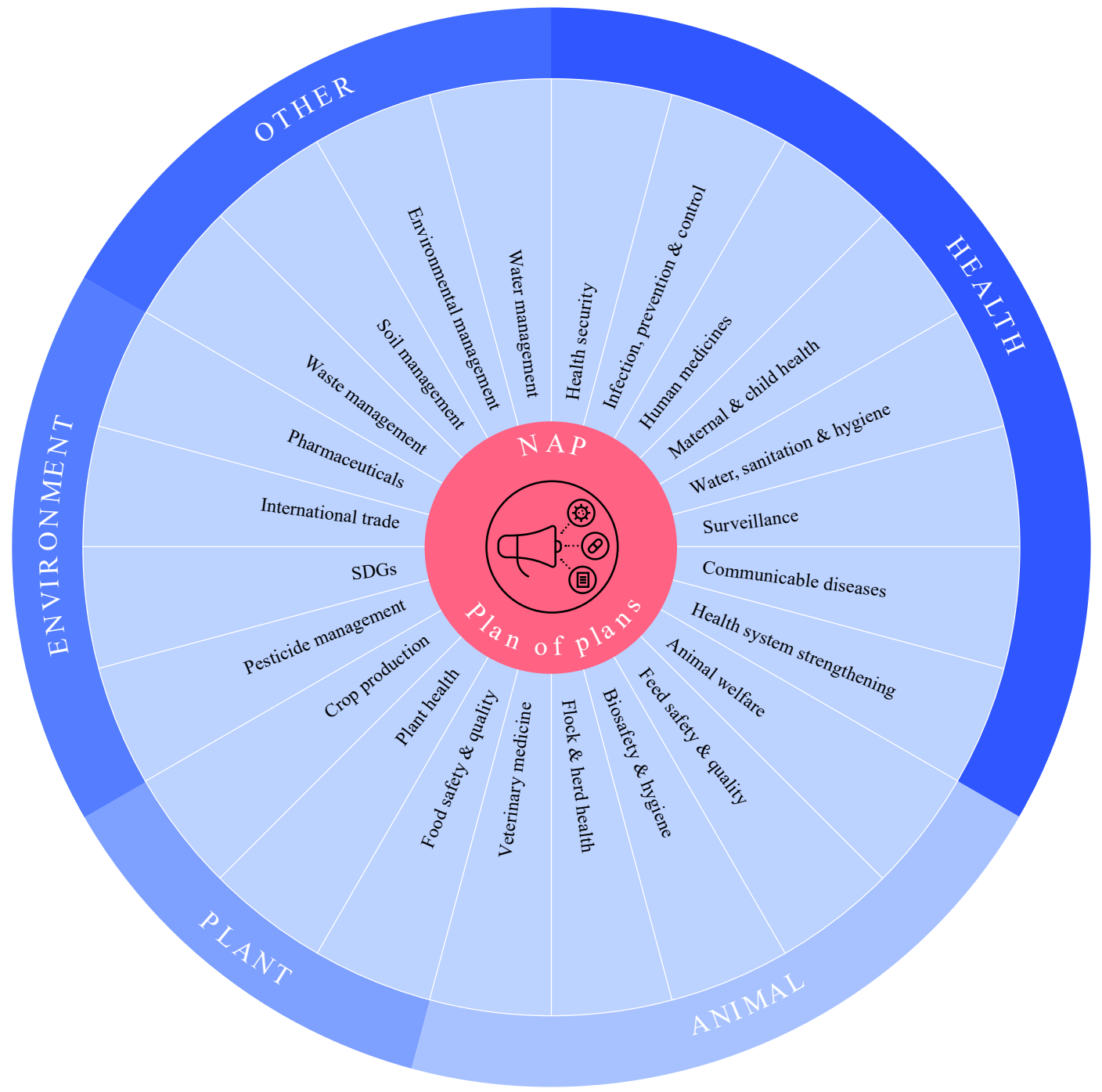

FIGURE 1. Turning plan into action: an overview for One Health approach [quoted from WHO (8)]. Abbreviation: SDGs=sustainable development goals; NAP=national action plans. 


\section{Theme - "Spread Awareness, Stop Resistance"}

As in previous years, the overall slogan for antimicrobial resistance awareness and WAAW is "Antimicrobials: Handle with Care." With the new theme of "Spread Awareness, Stop Resistance" in WAAW 2021 (1), we want to encourage stakeholders in human, animal, and environmental health to be "AMR Awareness" champions in their families, communities, and places of work. We hope everyone will engage with the global campaign activities and feel empowered to organize events to raise awareness as to what AMR is, and the actions of workplaces, communities, and/or government that can control the spread of AMR. We also hope you will share your personal stories so we can shift our thinking of AMR beyond the "drugs and bugs" narrative and ensure the consequences of AMR are effectively communicated to all the stakeholders. Different audiences will require different levels of detail delivered in comprehensible formats. The objective is for people everywhere to see, hear, relate to, and understand AMR as a health threat affecting our environment, animals, families, and communities and as relevant in our dayto-day lives. The big concern is that, if left unchecked, resistant microbes will develop with limited therapeutic options resulting in increased morbidity and mortality in both humans and animals and the associated costs to individuals and society. There is a limited number of new antibiotics in the research and development pipeline, therefore more powerful drugs are not the solution to the AMR crisis.

\section{Global Action Plan and the China National Action Plan}

In 2015, to advance the global and national response to AMR, the World Health Assembly issued resolution WHA68.7 calling for all Member States to develop AMR National Action Plans (NAPs) that address the 5 objectives of the Global Action Plan (GAP) by May 2017. The WHO GAP provides a framework to support countries in developing their NAPs. As of July 2021, 145 countries have developed NAPs and an additional 41 countries are currently developing a NAP. Among 145 countries, China has developed its own NAP with contributions from 14 multisectoral departments (5). In 2020, according to the Tripartite AMR Country SelfAssessment Survey data, approximately $20 \%$ of NAPs were fully funded and $40 \%$ had a budgeted operational plan $(6-7)$. Embedding AMR activities in government planning and budgeting processes at the national/subnational, sectoral, and departmental levels is one strategy for progress outlined in the draft WHO implementation handbook for NAPs on AMR (Figure 2). The cost of desired actions and a cost-benefit analysis can aid policymakers and implementers in prioritizing within resource-limited settings to revise plans, as deemed necessary (e.g., the number or scale of activities can be adjusted to fit available budgets). Additional information on selecting activities in AMR NAPs is available in converting plans into action for tackling AMR (Figure 3).

\section{Where to find the tool and who should use it}

The WHO costing and budgeting tool, accompanying documentation and resources are available on the WHO AMR website (8). The primary audience for the WHO tool is national AMR (One Health) stakeholders. Given that the technical expertise required to develop operational plans and budgeting processes is often ministry or sector specific, costing of individual activities and components might be conducted separately by individual ministries or sectors, and subsequently combined into a single costed plan (modular approach). Although specific processes might differ depending on the country, the following steps illustrate a possible coordination plan of costing across various actors and sectors:

- Step 1: The National AMR coordinating mechanism receives/downloads the tool and resources pack and designates a (or multiple) costing coordinator(s).

- Step 2: The costing coordinator reviews this user guide and available training materials and tests the tool functionality/features. If questions arise, the costing coordinator contacts the WHO helpdesk (amrnaphelpdesk@who.int) for additional technical assistance.

- Step 3: The costing coordinator, together with the members of the national AMR coordinating mechanism agree on the process for completing the costing tool: 1) one costing tool to be filled out for all activities irrespective of sector/ministry/department; or 2) each sector/ministry/department fills out the tool separately with the designated costing coordinator. Subsequently, a lead costing coordinator will compile the information into one costed plan (modular approach).

- Step 4: The costing coordinator holds a meeting with the relevant sectors, ministries and stakeholders who will implement the costed activities selected and prioritized. 


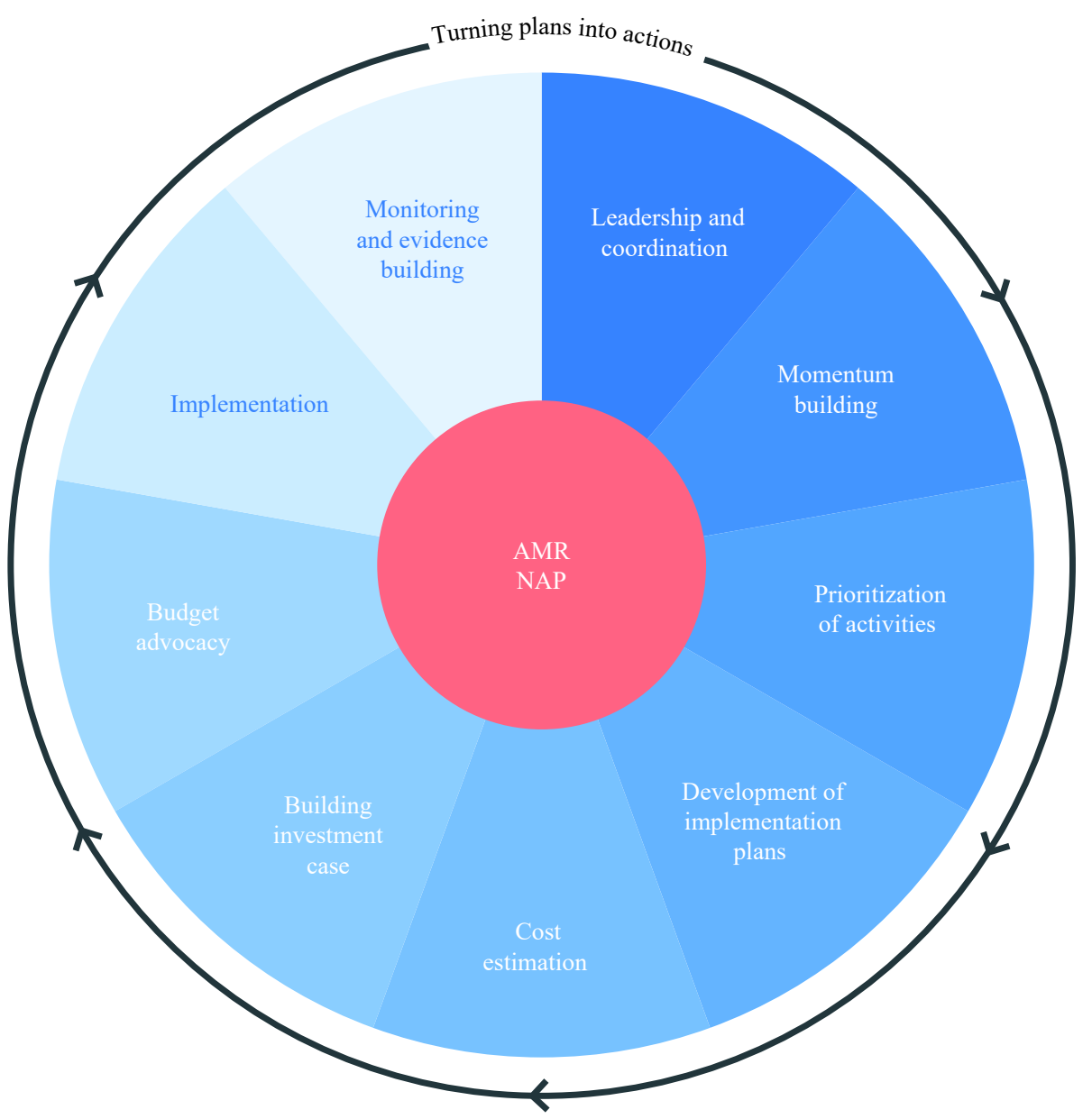

FIGURE 2. Overview of AMR NAP development and implementation [quoted from WHO (8)].

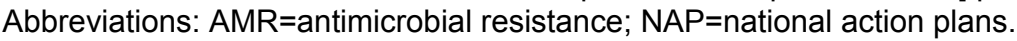

- Step 5: The costing coordinator completes the draft costing plan based on input from the relevant sectors, ministries, and stakeholders and forwards the completed costing tool to the main costing coordinator.

- Step 6: The costing coordinator reviews the submitted data and, if errors or incomplete data are detected, requests amendments.

- Step 7: The costing coordinators from the different sectors and ministries send revised, finalized results to the lead costing coordinator.

- Step 8: The main costing coordinator combines the data from multiple costing sheets to produce a comprehensive costed plan and dashboard (modular approach).

- Step 9: The costing coordinators share the comprehensive costed plan with the relevant actors.

\section{Overview and tool workflow completing the tool involves five key steps}

Figure 4 provides an overview of key components of the workflow. These steps are:

- Step 1. NAP entry. The user specifies the NAP priorities, objectives and activities that were selected for costing.

- Step 2. Basic inputs. The user enters key parameters relevant to their country, including ministry/implementer identifiers, funding opportunities, timelines to and during implementation, and unit costs for various items.

- Step 3. Costing matrix. These tabs are automatically generated after NAP entry is completed and are used to insert sub-activities and entering units and unit costs.

- Step 4. Funding (optional). The user enters existing funds from various sources/donors/stakeholders. This step is optional but recommended and can be completed at any time after the NAP entry is complete.

- Step 5. Dashboards. The user can specify various cross-tabulations and levels of analysis to produce dashboards and visualize all data. The Dashboard tab summarizes cost data entered by the user. The Funding Dashboard tab 


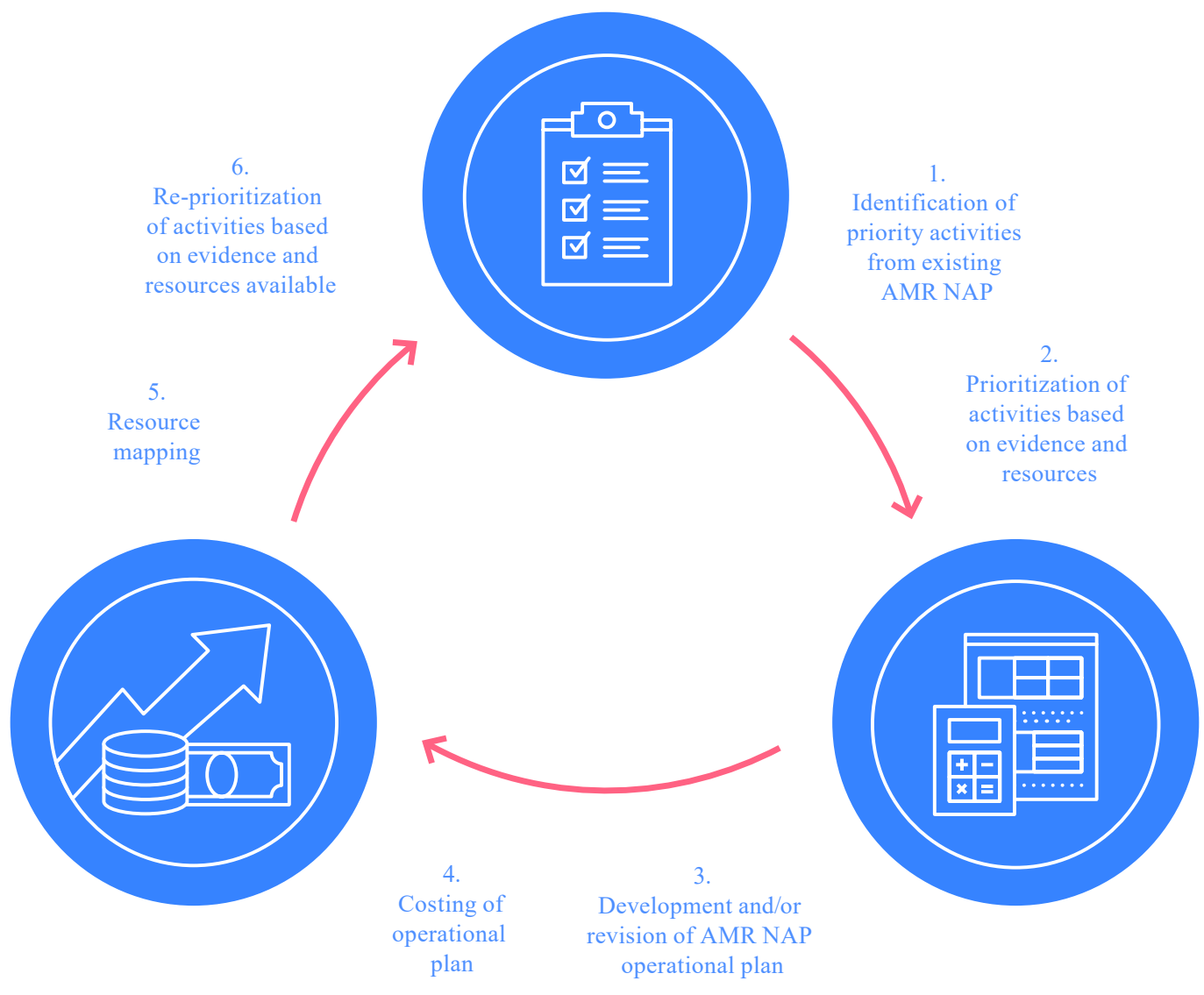

FIGURE 3. Activities' prioritization of NAP for AMR with One Health approach [quoted from WHO (8)].

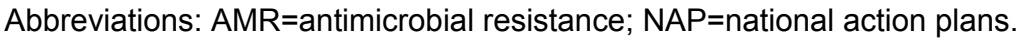

combines cost and funding data (if funding data in Step 4 above have been entered) by the user.

What China should do?

In the last decade China has positioned itself as global player in trade, commerce, and international engagement. China has also proven itself to be a world leader in understanding the perils of AMR, proceeded by swiftly producing and enforcing policy. First, by recognizing the emergence of AMR driven by using colistin in farming and subsequently enforcing its withdrawal (9). Second, in 2020, formally announcing the ban of all antibiotics in animal feeds across all meat production; by the end of 2025, large scale farms participant in action for reducing the use of veterinary antibiotics by more than $50 \%(10)$. AMR is a global challenge and will require global action to tackle it. Therefore, there is an urgent need for all countries to unite and work together in the war against AMR. Whilst COVID was an abrupt earthquake, AMR is a slow but massive tsunami that will engulf all people in all countries and its current trajectory is relentless. AMR's impact, in terms of morbidity and mortality and the financial impact on societies will dwarf that of coronavirus disease 2019 (COVID-19). Furthermore, as many different microbes are involved, it will not be readily remedied by vaccines. China is assisting in the global implementation programs by working closely, and collaborating, with international partners in tackling AMR, particularly in low-middle income countries through a spirit of trust and unity. AMR has no boundaries and is blind to creed, race, and social status. China is fortunate in having high caliber scientists in the areas of microbial genetics and human and animal health who are collaborating with international colleagues and thus, China is playing a pivotal role in our global fight against our common enemy.

Funding: National Nature Science Foundation of China (NSFC) project 216812303 and Newton International Fellowship NIF|R1\192293 of Royal Society.

doi: $10.46234 / \mathrm{ccdcw} 2021.241$ 


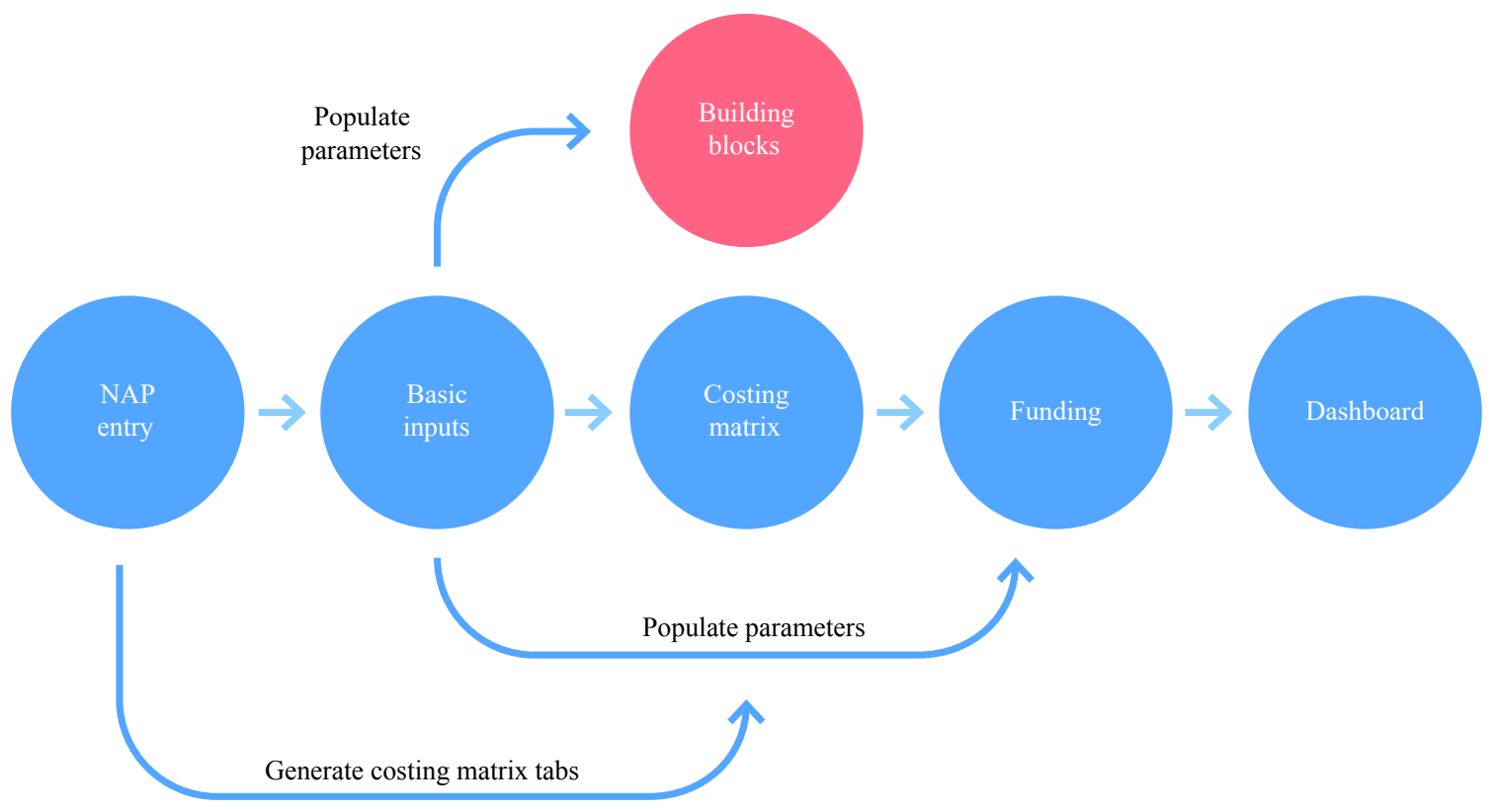

FIGURE 4. Tool workflows for NAP: from plan to action [quoted from WHO (8)]. Abbreviation: NAP=national action plans.

\# Corresponding authors: Timothy Walsh, timothy.walsh@zoo.ox.ac.uk; Yongning Wu, wuyongning@cfsa.net.cn.

\begin{abstract}
${ }^{1}$ Institute for Global Food Security, Queen's University of Belfast, Belfast, United Kingdom; ${ }^{2}$ Ineos Oxford Institute for Antimicrobial Research, University of Oxford, Oxford, United Kingdom; ${ }^{3}$ NHC Key Laboratory of Food Safety Risk Assessment, Chinese Academy of Medical Science Research Unit (2019RU014 Food Safety), China National Center for Food Safety Risk Assessment, Beijing, China.
\end{abstract}

Submitted: November 09, 2021; Accepted: November 15, 2021

\title{
REFERENCES
}

1. World Health Organization. World antimicrobial awareness week 2021 - spread awareness, stop resistance. 2021. https://www.who.int/news/item/26-082021-world-antimicrobial-awareness-week-2021-spread-awareness-stop-resistance. [2021-11-8].

2. World Health Organization. World antimicrobial awareness week: campaign Guidanc. 2021. https://www.who.int/publications/m/item/worldantimicrobial-awareness-week-campaign-guidance. [2021-11-8].

3. World Health Organization, Food and Agriculture Organization, World Organisation for Animal Health, UN Environment Programme. Antimicrobial resistance and the United Nations sustainable development cooperation framework: guidance for United Nations country teams. 2021. https://www.who.int/publications/i/item/9789240036024. [2021-11-8].

4. Wu D, Elliott C, Wu YN. Food safety strategies: the one health approach to global challenges and China's actions. China CDC Wkly 2021;3(24):507 13. http://dx.doi.org/10.46234/ccdcw2021.131.

5. Xiao YH, Li LJ. China's national plan to combat antimicrobial resistance. Lancet Infect Dis 2016;16(11):1216 - 8. http://dx.doi.org/10.1016/S1473-3099 (16)30388-7.

6. World Health Organization. Global database for the tripartite antimicrobial resistance (AMR) country self-assessment survey (TrACSS). Geneva: World Health Organization. 2018. https://amrcountryprogress.org/. [2021-11-8].

7. World Health Organization. Turning plans into action for antimicrobial resistance (AMR). Working paper 2.0: implementation and coordination. Geneva: World Health Organization. 2019. https://www.who.int/antimicrobial-resistance/publications/AMR-Turning-plans-into-action-working-paper-march2019.pdf?ua $=1$. [2021-11-8].

8. World Health Organization. WHO costing and budgeting tool for national action plans on antimicrobial resistance: user guide. Geneva: World Health Organization. 2021. https://www.who.int/publications/i/item/9789240036901. [2021-11-8].

9. Walsh TR, Wu YN. China bans colistin as a feed additive for animals. Lancet Infect Dis 2016;16(10):1102 - 3. http://dx.doi.org/10.1016/S1473-3099(16) 30329-2.

10. Ministry of Agriculture and Rural Affaires of the People's Republic of China. National action plan for reducing the use of veterinary antibiotics (20212025). 2021. http://www.moa.gov.cn/govpublic/xmsyj/202110/t20211025_6380448.htm. [2021-11-8]. (In Chinese). 


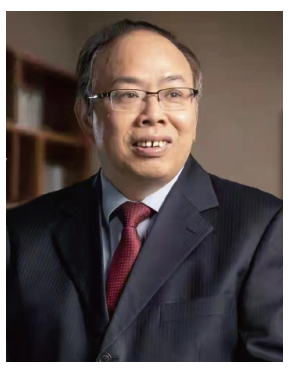

Yongning $\mathrm{Wu}, \mathrm{PhD}, \mathrm{MD}$

Chief Scientist and Professor, China National Center for Food Safety Risk Assessment, Beijing, China

Director of NHC Key Laboratory of Food Safety Risk Assessment and Chinese Academy of Medical Science Research Unit (2019RU014 Food Safety), Beijing, China

Member of the WHO Strategic and Technical Advisory Group for Antimicrobial Resistance (STAG-AMR 2019-2020)

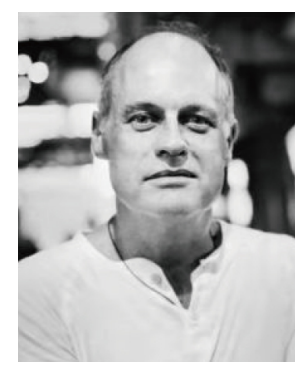

Timothy Walsh, PhD, MD

Professor of Medical Microbiology and Antibiotic Resistance, Department of Zoology, University of Oxford, Oxford, UK

O.B.E for Microbiology and International Development, UK

Advisor to the China CDC, Beijing, China

Member of the WHO Strategic and Technical Advisory Group for Antimicrobial Resistance (STAG-AMR 2021-2022)

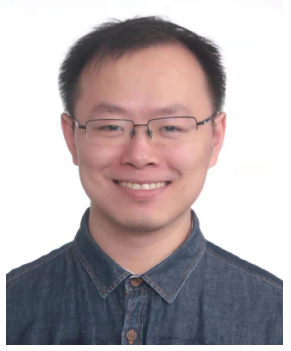

$\mathrm{Di} \mathrm{Wu}, \mathrm{PhD}$

Newton International Fellow of Royal Society, London, UK

Institute for Global Food Security, Queen's University of Belfast, Belfast, UK

School of Biological Sciences, Queen's University of Belfast, Belfast, UK 